\title{
Prediction of BLEVE Mechanical Energy by Implementation of Artificial Neural Network
}

\author{
Behrouz Hemmatian $^{1}$, Joaquim Casal ${ }^{2}$, Eulàlia Planas ${ }^{2}$, Behnam Hemmatian $^{3}$, Davood Rashtchian H,* $^{1, *}$ \\ ${ }^{1}$ Center for Process Design, Safety and Loss Prevention (CPSL), Chemical and Petroleum Engineering \\ Department, Sharif University of Technology, Tehran, Iran \\ ${ }^{2}$ Centre for Technological Risk Studies (CERTEC), Barcelona East School of Engineering (EEBE), \\ Universitat Politècnica de Catalunya, BarcelonaTech (UPC), Eduard Maristany 10-14, 08019-Barcelona, \\ Catalonia, Spain \\ ${ }^{3}$ Department of Electrical Engineering, Amirkabir University of Technology (AUT), 424 Hafez Ave, \\ Tehran, Iran
}

\begin{abstract}
In the event of a BLEVE, the overpressure wave can cause important effects over a certain area. Several thermodynamic assumptions have been proposed as the basis for developing methodologies to predict the mechanical energy associated in such a wave and the peak overpressure. According to a recent comparative analysis, methods based on real gas behavior and adiabatic irreversible expansion assumptions can give a good estimation of this energy. In this communication, the Artificial Neural Network (ANN) approach has been implemented to predict the BLEVE mechanical energy for the case of propane and butane. Temperature and vessel filling degree at failure have been considered as input parameters, and the BLEVE blast energy has been estimated as output data by the ANN model. A Bayesian Regularization algorithm was chosen as the three-layer backpropagation training algorithm. Based on the neurons optimization process, the number of neurons at the hidden layer was five in the case of propane and four in the case of butane. The transfer function applied in this layer was a sigmoid, because it had an easy and straightforward differentiation for using in the backpropagation algorithm. For the output layer, the number of neurons had to be one in both cases, and the transfer function was purelin (linear). The model performance has been compared with experimental values proving that the mechanical energy of a BLEVE explosion can be adequately predicted with the Artificial Neural Network approach.
\end{abstract}

\section{Keywords}

BLEVE; vessel explosion; explosion energy; blast overpressure; pressure wave; Artificial Neural Network.

\footnotetext{
* Corresponding author:

E-mail address: rashtchian@sharif.edu (D. Rashtchian)
} 


\section{Introduction}

BLEVEs are strong explosions associated to the catastrophic failure of a vessel containing a liquid plus vapor at a temperature significantly higher than its boiling temperature at atmospheric pressure; the instantaneous increase in volume of both liquid and vapor generates an overpressure wave which can be very strong. This wave can be attributed to the liquid vaporization, to the expansion of the pre-existent vapor or to the added effect of both phenomena, this later approach being the most conservative.

In order to calculate the effects and consequences of this overpressure, the mechanical energy associated to it must be known."

There are several ways to calculate BLEVE mechanical energy, which are based on different thermodynamic assumptions:

- Constant volume energy addition (Brode,1959)

- Ideal gas behavior and isentropic expansion (Prugj, 1991)

- Thermodynamic availability (Crowl, 1991, 1992)

- Isothermal expansion (Smith et al., 1996).

These methods take into account the contribution of both the liquid vaporization and the preexisting vapor expansion. Furthermore, another approach has been proposed (Casal and Salla, 2006, based on the superheating energy SE) which takes into account only the vaporization of the liquid. According to a comparative analysis (Hemmatian et al., 2017a), all the methods based on the assumptions above give rather conservative results, but the methods based on real gas behavior and adiabatic irreversible expansion give more realistic values. Following a relatively different approach (Hemmatian et al., 2017b), the mechanical energy can be estimated as a function of the vessel filling degree and temperature at the rupture moment. In practice, however, it should be taken into account that only part of the mechanical energy is invested in creating the overpressure. The other part is used for breaking the vessel and fragmentation. In the case of ductile failure, approximately $40 \%$ of the mechanical energy is converted to blast. However, this number could vary between $40 \%$ and $50 \%$ depending on the circumstances.

From the point of view of the emergency management, a BLEVE can occur at any moment depending on the circumstances when a vessel is under the effects of fire (Hemmatian et al., 2015). For example, the first BLEVE in San Juanico accident (1984) happened after 70 seconds of the first explosion (which probably originated by jet fires). However, the time to failure can be more prolonged: for example, it has been reported that an LPG tanker BLEVEd after 70 minutes of being exposed to fire (Planas et al., 2015). Anyway, the prediction of BLEVE mechanical energy is quite important to define the possible effects and the safe distances.

Some of the existing methods require complex thermodynamic information and calculations. A different approach that can help in solving this problem could be that based on the Artificial Neural 
Network (ANN), used nowadays as a handy toolkit for the simulation, prediction, and modeling of various engineering and scientific problems.

In this study, ANN approach has been implemented to produce a function for calculating the BLEVE mechanical energy, specifically for propane and butane as the most common substances involved in BLEVE accidents (Hemmatian et al., 2019). The function thus obtained only depends on the rupture temperature and vessel filling level. The values thus obtained have been compared with some experimental data taken from the literature.

\section{Theory and methods}

\subsection{Dataset preparation}

Two datasets for propane and butane were prepared for the ANN approach by using a method based on real gas behavior and adiabatic irreversible expansion (Planas Cuchi et al., 2004). The required thermodynamic data were obtained from NIST Reference Fluid Thermodynamic and Transport Properties Database (REFPROP) version 9.1. (Lemmon et al., 2007). Temperature (K), filling level $(\%)$ and mechanical energy $\left(\mathrm{MJ} / \mathrm{m}^{3}\right)$ were registered in the datasets. The database interval was designed for filling level ranging between $1 \%$ and $90 \%$ in the case of propane, and between $1 \%$ and $99 \%$ for butane. The initial temperatures were $300 \mathrm{~K}$ and $283 \mathrm{~K}$ for both propane and butane. The ANN was trained in those aforementioned intervals. Propane and butane datasets had 121 and 201 data points, respectively. Those datasets were used in the ANN for generating the related functions. Finally, the resulting functions were applied to the two sets of experiments to analyze the goodness of the method.

\subsection{BLEVE experimental data}

Two sets of experimental data were used to check the models' performances. In Johnson experiments (Johnson et al., 1990; Laboureur et al., 2014), there are 7 BLEVE data obtained by using butane and propane. (Table 1) The heating of the contained liquid was applied with electric immersion. The vessels were ruptured by the detonation of a linear explosive. The overpressure was measured at 25, 50, 100 and 150 meters away.

Table 1. Johnson experiments (Johnson et al., 1990; Laboureur et al., 2014)

\begin{tabular}{cccccc}
\hline Johnson & Fluid & $\mathbf{m}(\mathbf{k g})$ & $\mathbf{V}\left(\mathbf{m}^{\mathbf{3}}\right)$ & $\mathbf{P}_{\text {rupt }}(\mathbf{k P a})$ & $\begin{array}{c}\text { Filling Level } \\
\mathbf{( \% )}\end{array}$ \\
\hline $\mathbf{1}$ & Butane & 2000 & 5.659 & 1460 & 75 \\
$\mathbf{2}$ & Butane & 2000 & 5.659 & 1510 & 76 \\
$\mathbf{3}$ & Butane & 1000 & 5.659 & 1520 & 38 \\
$\mathbf{4}$ & Butane & 2000 & 5.659 & 770 & 68 \\
$\mathbf{5}$ & Butane & 2000 & 10.796 & 1510 & 40 \\
$\mathbf{6}$ & Propane & 2000 & 5.659 & 1520 & 77
\end{tabular}


9 fired propane BLEVE experiments were reported by Birk et al. (Birk and VanderSteen, 2006; Birk et al., 2007). The overpressure was measured at 10, 20, 30 and 40 meters away. (Table 2)

Table 2. Birk experiments (Birk and VanderSteen, 2006; Birk et al., 2007)

\begin{tabular}{llcccc}
\hline Birk & Fluid & $\mathbf{m}(\mathbf{k g})$ & $\mathbf{V}\left(\mathbf{m}^{\mathbf{3}}\right)$ & $\mathbf{P}_{\text {rup }}(\mathbf{k P a})$ & Filling Level (\%) \\
\hline $\mathbf{1}$ & Propane & 150 & 2 & 1863 & 17 \\
$\mathbf{2}$ & Propane & 309.3 & 2 & 1846 & 35 \\
$\mathbf{3}$ & Propane & 117 & 2 & 1699 & 13 \\
$\mathbf{4}$ & Propane & 184.6 & 2 & 1894 & 21 \\
$\mathbf{5}$ & Propane & 116.9 & 2 & 1573 & 12 \\
$\mathbf{6}$ & Propane & 453.1 & 2 & 1803 & 51 \\
$\mathbf{7}$ & Propane & 475.1 & 2 & 1563 & 52 \\
$\mathbf{8}$ & Propane & 470.3 & 2 & 1813 & 53 \\
$\mathbf{9}$ & Propane & 538.4 & 2 & 1858 & 61 \\
\hline
\end{tabular}

\subsection{Artificial Neural Network (ANN)}

Neurons are the basic elements of the biological neural network in the human brain. A neuron receives data from neighboring neural cells through dendrites and makes some process in the soma (body), transferring a signal to the next neuron through the axon; data transfer is performed by synapses through electrochemical signals. (Yadav et al., 2014)

The Artificial Neural Network is a method that can be applied to analyze and calculate data for different problems and solve them with the same pattern as that of a biological neural network. The first attempts in this field were due to Hebb in the 1940s (Hebb, 1949). After that, some researchers such as Hopfield, Rumelhart, Grossberg, and Widrow developed this method in the 1980s. (Hopfield, 1982; Rumelhart et al., 1986; Grossberg,1982; Widrow et al., 1987)

With ANN, complicated problems could be solved through parallel and distributed processing. It solves problems without requiring too complicated formulation; because of this, it could save time significantly in comparison with closed-form solving methods. Moreover, this methodology enables to approximate any non-linear function to a compact set of data with a specified accuracy (Siddique and Adeli, 2013).

Every neuron model consists of a segment that signals (i.e., input variables) import through it and acts like a synapse. At first, each one of the inputs $\left(x_{i}\right)$ is multiplied by its corresponding weight 
value " $\boldsymbol{w} "$. After the summation of these values, a bias value " $b$ " can be added to the result. A summary of this process for $n$ inputs is shown in equation (1):

$$
n e t=\left(\sum_{i=1}^{n} \omega_{i} x_{i}\right)+b
$$

At the end of this process, the result enters in a transfer function (i.e., $f$ ) and give output values (i.e., y) (Equation (2)):

$$
y=f(n e t)
$$

The usual forms of the transfer function are linear, step, ramp or sigmoid. Figure 1 shows the structure of an ANN.
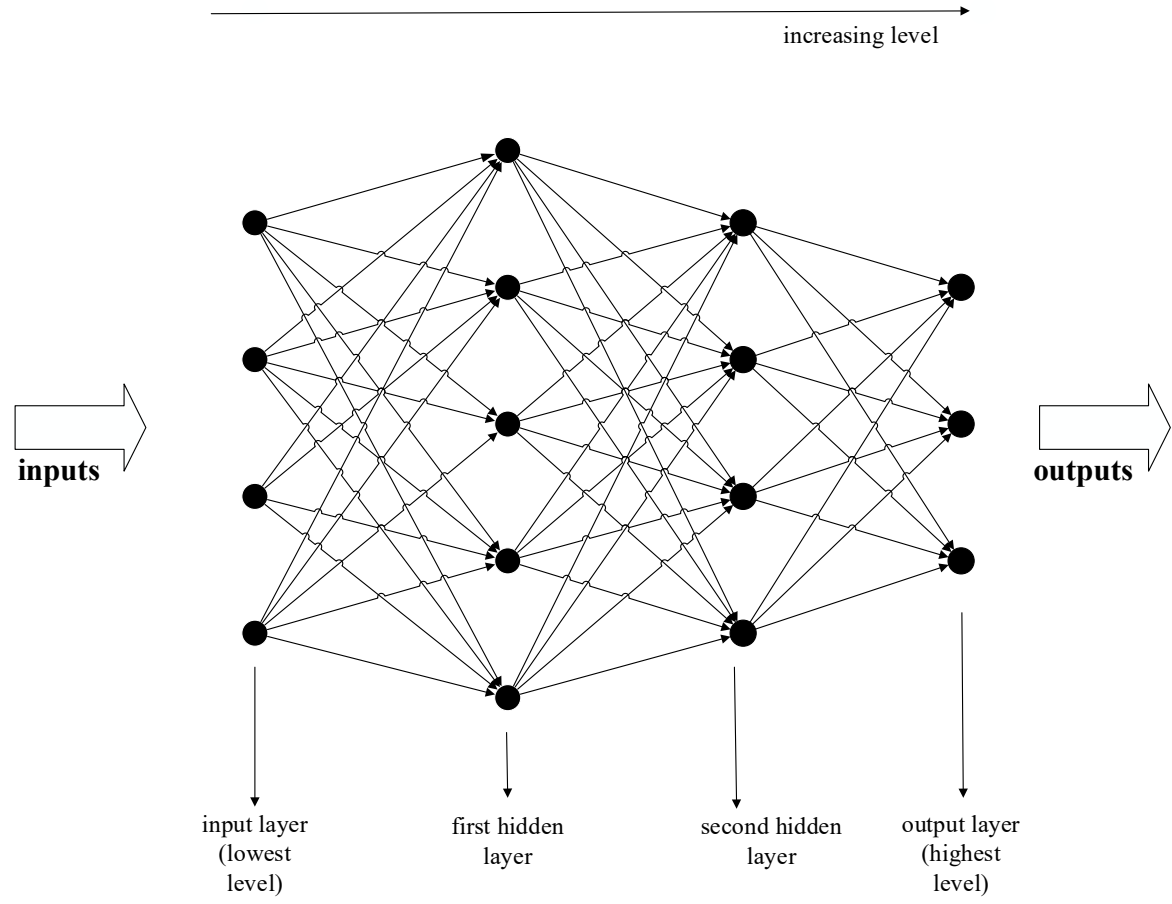

Figure 1. Structure of an Artificial Neural Network. (Siddique and Adeli, 2013)

Some neurons connect, forming a layer of neurons. A network includes one or more of these layers. According to the configuration and the way of connection between neurons, there can be different types of neural networks. Generally, they can be separated into two categories: 1) feedforward neural networks and 2) feedback neural networks. 


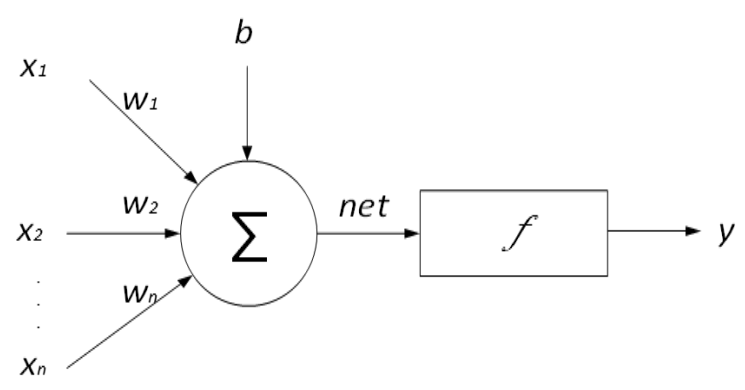

Figure 2. Feedforward Neural Network.

In a feedforward neural network, the signals travel in a forward way, they cannot come back, and there is no feedback. This type of neural network (e.g., Figure 2) can be represented in vector form by equation (3):

$$
Y=f(W \cdot x+\mathrm{b})
$$

Here, $Y$ is the output vector, $W$ is the weight matrix, $\mathrm{b}$ is a bias vector and $f$ is a transfer function.

The primary task of the neural network is to define the weights and biases in a way that adapts the output to the inputs with a minimum error. A training process does the modification of weights and biases. The training method used for solving the network in this work has been the Bayesian Regulation method, which can be applied to feedforward neural networks training. It is based on a statistical approach and assumes that the values of weights and biases are related to a distribution function with unknown variance. The primary task is to estimate the parameters using statistical techniques (Siddique and Adeli, 2013; Foresee and Hagan, 1997; Nguyen, 1998).

\section{Results and discussion}

\subsection{Backpropagation training algorithm}

Three different backpropagation (BP) algorithms, i.e., Levenberg - Marquardt (Hagan and Menhaj, 1994), Bayesian Regularization (MacKay, 1992; Ticknor, 2013) and Scaled Conjugate Gradient (Møller, 1993), were studied to find the best one for the ANN. Five neurons were considered for the comparative study and, finally, the algorithm with the lowest mean square error (MSE) was chosen as the BP algorithm. As it is shown in Table 3 and Table 4, the Bayesian Regularization algorithm has the lowest MSE for propane and butane. Therefore it was the one selected in this study. The MSE values for these two substances were $2.29 \cdot 10^{-4}$ and $4.63 \cdot 10^{-5}$, respectively.

Table 3. Backpropagation training algorithms for propane

\begin{tabular}{lcccc}
\hline $\begin{array}{l}\text { Backpropagation } \\
\text { algorithm }\end{array}$ & Function & $\begin{array}{c}\text { Testing } \\
\text { Mean square } \\
\text { error (MSE) }\end{array}$ & Epoch* $^{\text {Regression }}$ & Best linear equation \\
& & R value & \\
\hline
\end{tabular}




\begin{tabular}{lccccc}
\hline $\begin{array}{l}\text { Levenberg- } \\
\text { Marquardt } \\
\text { backpropagation }\end{array}$ & trainlm & 0.000364 & 232 & 0.99996 & Output $=1 \cdot$ Target +0.0049 \\
\hline $\begin{array}{l}\text { Bayesian } \\
\text { Regularization }\end{array}$ & trainbr & 0.000229 & 385 & 0.99998 & Output $=1 \cdot$ Target \\
+0.00098 \\
\hline $\begin{array}{l}\text { Scaled Conjugate } \\
\text { Gradient }\end{array}$ & trainscg & 0.0736 & 39 & 0.99205 & Output $=0.97 \cdot$ Target \\
+0.17
\end{tabular}

*In training process, each training level names as "training epoch". All the inputs are entered in each training epoch and give outputs which compared with target to give the error. By this process, weights and biases are calculated and modified at each epoch.

Table 4. Backpropagation training algorithms for butane

\begin{tabular}{lccccc}
\hline $\begin{array}{l}\text { Backpropagation } \\
\text { algorithm }\end{array}$ & Function & $\begin{array}{l}\text { Testing } \\
\text { Mean square } \\
\text { error (MSE) }\end{array}$ & $\begin{array}{c}\text { Epoch } \\
\text { Regression } \\
\text { R value }\end{array}$ & Best linear equation \\
\hline $\begin{array}{l}\text { Levenberg- } \\
\begin{array}{l}\text { Marquardt } \\
\text { backpropagation }\end{array}\end{array}$ & trainlm & $5.85 \cdot 10^{-5}$ & 116 & 1 & Output $=1 \cdot$ Target +0.0019 \\
$\begin{array}{l}\text { Bayesian } \\
\text { Regularization }\end{array}$ & trainbr & $4.63 \cdot 10^{-5}$ & 1000 & 0.99999 & Output $=1 \cdot$ Target -0.0012 \\
$\begin{array}{l}\text { Scaled Conjugate } \\
\text { Gradient }\end{array}$ & trainscg & $8.39 \cdot 10^{-1}$ & 17 & 0.94398 & Output $=0.93 \cdot$ Target +0.33 \\
\hline
\end{tabular}

\subsection{Optimizing neurons number}

The determination of the neurons' number is essential. Considering few neurons causes underfitting and, contrarily, overfitting can occur if the number of neurons is higher than a specific value. In this study, an ANN was trained based on Bayesian Regularization BP algorithm for propane and butane. The number of neurons was varied from 1 to 20, and an optimum number of neurons was chosen based on the minimum value of MSE of the training. According to Figure 3, the optimum number of neurons for propane and butane were five and four, respectively. The MSE on the minimum value of MSE for propane and butane were 0.00025 and 0.000129 , respectively, which were lower than 0.001 as a sufficient threshold value for MSE. 
a)
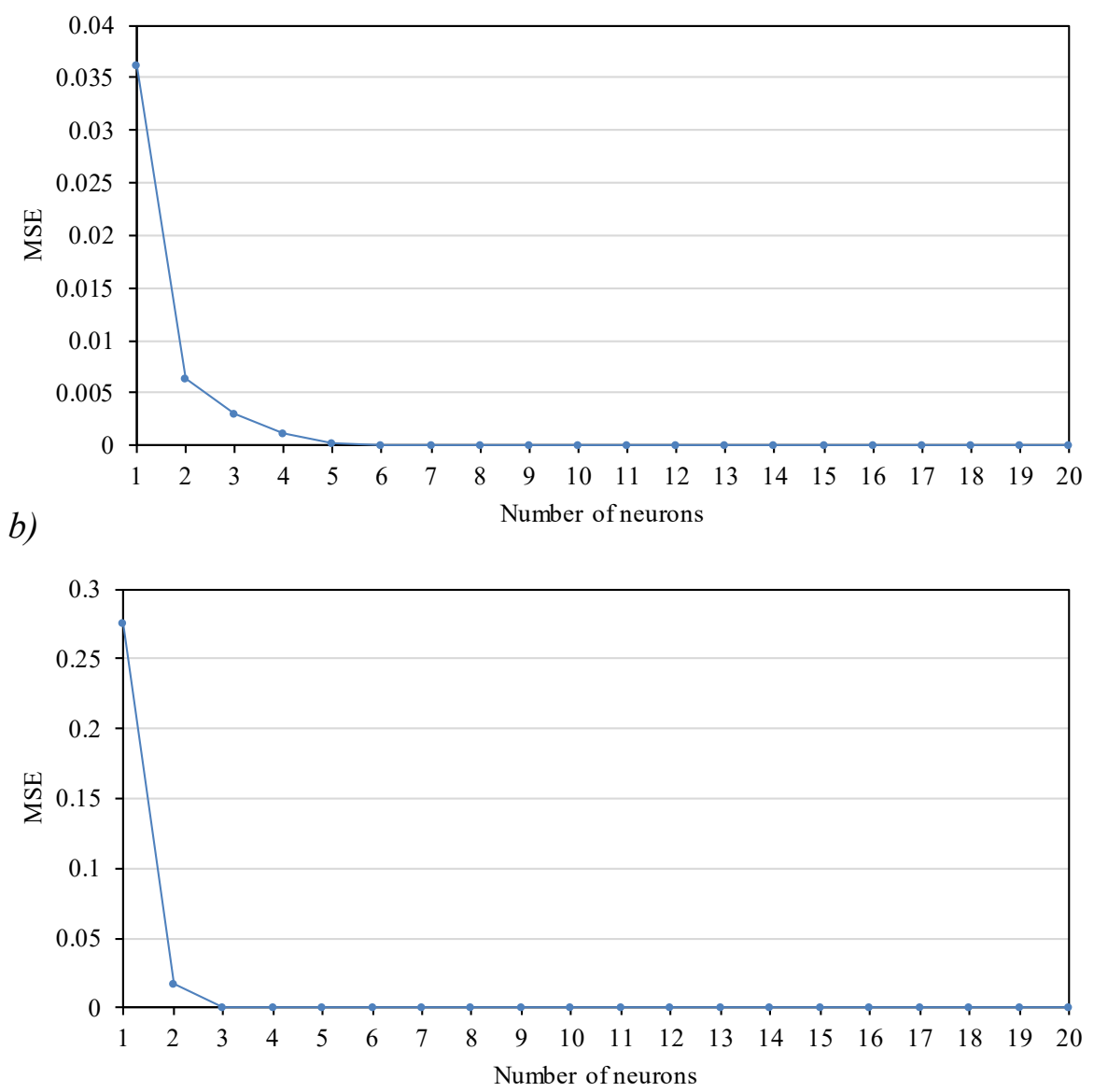

Figure 3. Optimized neurons number for: a) propane, b) butane.

\subsection{ANN setup and results}

For solving the problem, a multilayer feed forward neural network was used. A general picture of this method has been depicted in Figure 4. The multilayer feed forward network consists of three layers. The first one is the input layer through which the data (i.e., filling level and temperature just before the explosion as input parameters) are imported into the network, and the last layer is the output one (i.e., mechanical energy per cubic meter of the vessel as output data), which gives the target data. Between these two mentioned layers, there is another hidden layer. The number of hidden layers depends on the accuracy that is required for a particular problem. In this analysis, the number of hidden layers was set to one, which provided reasonable accuracy. Based on the neurons optimization process, the number of neurons at the hidden layer was five in the case of propane and four in the case of butane. The transfer function applied in this layer was a sigmoid because it had an easy and straightforward differentiation for using in the backpropagation 
algorithm. For the output layer, the number of neurons had to be one in both cases, and the transfer function was purelin (linear).

a)

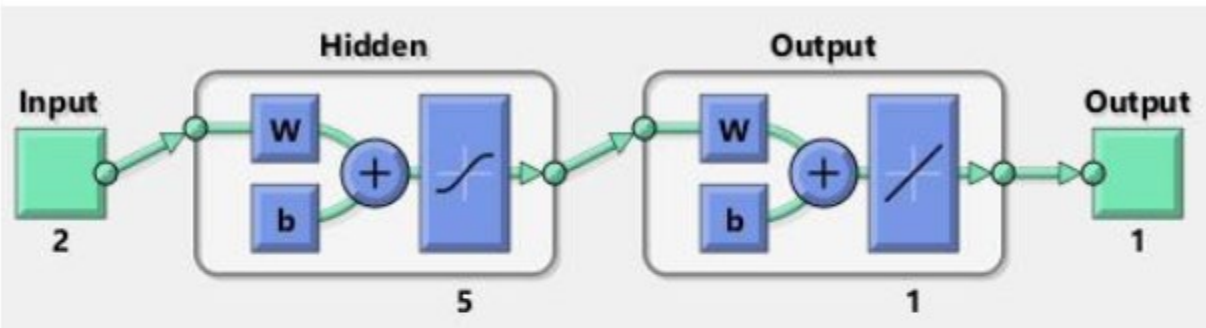

b)

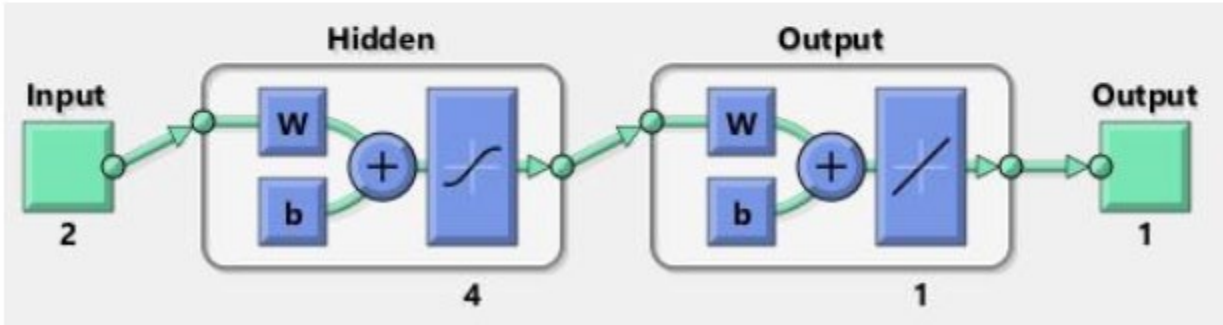

Figure 4. Artificial Neural Network structure for: a) propane, b) butane.

The process was performed with the MATLAB Neural Network toolbox version R20015a (8.5.0.197613 - License Number: 107001). The number of input data for the training process was 121 and 201 for propane and butane, respectively. It reached a convergence level after 348 iterations in the case of propane and 191 iterations with butane. The validation process was done with $15 \%$ of the data to check the network generalization. The resulting network was tested with $15 \%$ of the data to provide an independent measure of the network performance during and after training. In the training process, a part of the available data was used to train the network and find out the suitable weights and biases. These quantities were checked in the validation process. Based on the obtained results, it would be preferred to choose $15 \%$ of data randomly, which finally gave good results. The obtained results also demonstrated a good level of performance according to the prepared dataset. (Beale et al., 2010)

For propane, the designed network regression $\mathrm{R}$ values were close to one, showing that there was a close correlation between ANN outputs and target values from prepared dataset. Figure 5 shows how much accurate the ANN model is. 

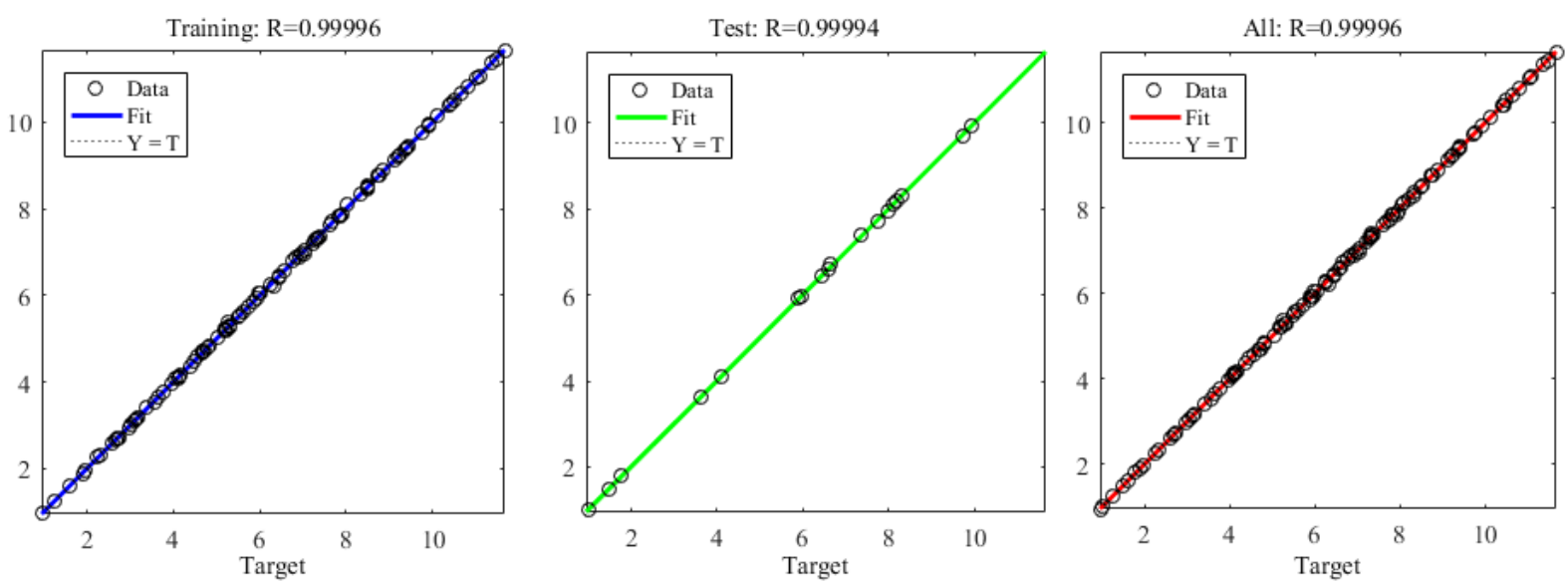

Figure 5. Regression plots of the mechanical energy (MJ) per cubic meter of the vessel for propane; left: during training; middle: during the testing; right: all the data including validation; Abscissa: target values from prepared dataset - Ordinate: ANN outputs.

Also, the optimized weights for propane produced by the artificial neural network model are summarized in Table 5.

Table 5. Propane's weights matrix

\begin{tabular}{lccc}
\hline \multirow{2}{*}{ Neuron } & \multicolumn{2}{c}{ W1 } & W2 \\
\cline { 2 - 4 } & \multicolumn{2}{c}{ Input variables } & Output \\
\cline { 2 - 4 } & Temperature & Filling level & Energy \\
\hline 1 & -1.2335 & -0.2862 & -0.8641 \\
2 & 1.7512 & 0.3928 & 0.5474 \\
3 & -0.8560 & 0.2377 & 3.16 \\
4 & 1.2560 & -0.3420 & 1.1380 \\
5 & 1.1617 & 0.2995 & 1.1103 \\
\hline W1: Weights between input and hidden layers & & \\
W2: Weights between hidden and output layers & &
\end{tabular}

In the case of butane, the regression results (Figure 6) show that the network was appropriately trained and a linear relation exists between output and target data. $R$ values were close to unity which indicated linearity between target values from prepared dataset and ANN output data. 

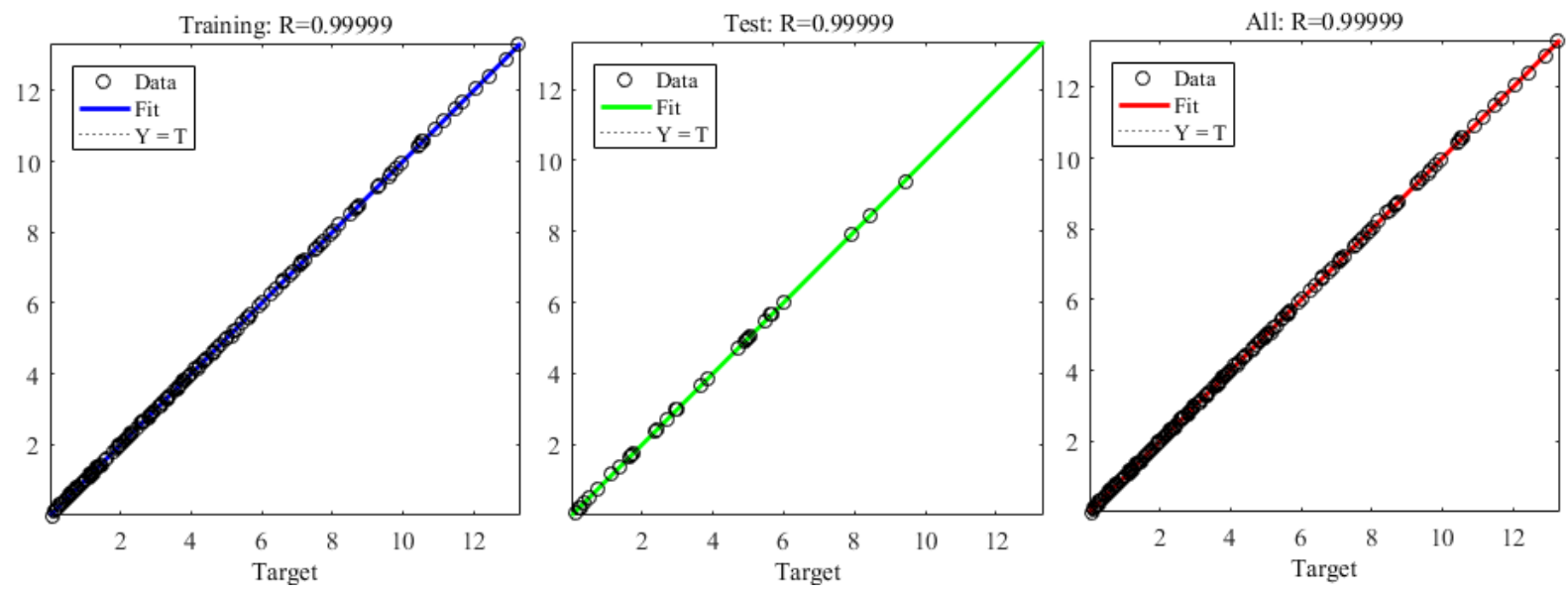

Figure 6. Regression plots of the mechanical energy (MJ) per cubic meter of the vessel for butane; left: during training; middle: during the testing; right: all the data including validation;

Abscissa: target values from prepared dataset - Ordinate: ANN outputs.

The optimal weights for butane from the artificial neural network between input and hidden layers (W1) and between hidden and output layers (W2) are shown in Table 6.

Table 6. Butane's weights matrix

\begin{tabular}{|c|c|c|c|}
\hline \multirow{3}{*}{ Neuron } & \multicolumn{2}{|c|}{ W1 } & \multirow{3}{*}{$\begin{array}{c}\text { W2 } \\
\text { Outpu } \\
\text { Energy }\end{array}$} \\
\hline & \multicolumn{2}{|c|}{ Input variables } & \\
\hline & Temperature & Filling level & \\
\hline 1 & -1.3757 & -0.4138 & -0.2668 \\
\hline 2 & 0.7913 & -0.1637 & -1.8164 \\
\hline 3 & -0.8212 & -0.1932 & -2.4487 \\
\hline 4 & -1.5521 & 0.4522 & -0.3672 \\
\hline
\end{tabular}

W1: Weights between input and hidden layers

W2: Weights between hidden and output layers

\subsection{Comparative study}

Root-Mean-Square Deviation (RMSD) (Piñeiro et al., 2008) as a statistical toolkit was used in the comparison of the results obtained with the ANN method and the aforementioned experimental data from Birk et al. (2006 and 2007) and Johnson et al. (1990). TNT equivalent mass and the well-known plot of the scaled distance vs. peak overpressure were utilized for obtaining the resulting overpressures $(\Delta \mathrm{P})$ at different distances. The results are shown in Table 7 , together with those obtained from polynomial expressions (Hemmatian et al., 2017b). As can be seen, the functions derived from the ANN show a good performance. It can be stated that both methods proposed are accurate and practical to be applied. The nonlinear relation between temperature and 
filling level (Hemmatian et al., 2017b) is probably the reason for the observed deviation that, as seen in the comparative analysis, remains in the range of the expected accuracy of this type of calculation and, therefore, should be considered acceptable. Figure 7 shows the differences between the various approaches and the experimental values. As can be seen, at short distances (corresponding to high overpressures) the calculated values from the four approaches are higher than the experimental ones. This should be attributed to the use of the TNT equivalency method for the estimation of the peak overpressure: this method gives usually better results in the far field, while it often overpredicts them in the near field.

Table 7. RMSD values for the comparison of $\Delta \mathrm{P}$ experimental values and the prediction from the different methodologies and the TNT equivalency method

\begin{tabular}{lcccc}
\hline \multicolumn{4}{c}{ Thermodynamic assumption* } \\
\hline RMSD & RAIE & SE & $\begin{array}{c}\text { Polynomial } \\
\text { approach }\end{array}$ & $\begin{array}{c}\text { Neural Net Fitting } \\
\text { approach }\end{array}$ \\
\hline Johnson-TNT eq. method & 2.3 & 2.2 & 2.2 & 2.2 \\
Birk-TNT eq. method & 4.9 & 4.1 & 4.2 & 4.3 \\
\hline
\end{tabular}

*RAIE = Real gas behavior and adiabatic irreversible expansion; SE = Liquid superheating energy 


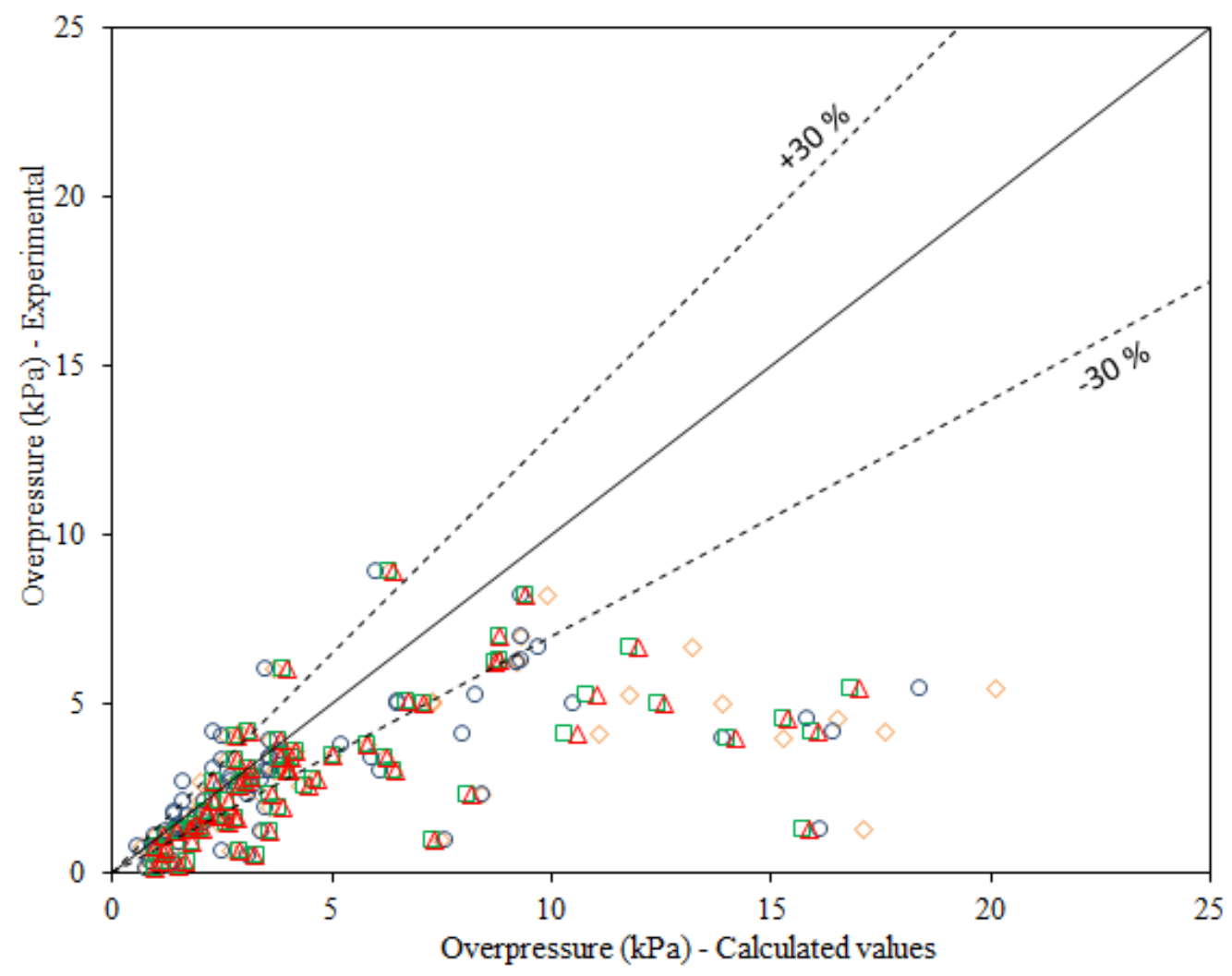

$\begin{array}{ll}\text { Real gas behavior and adiabatic irreversible expansion } & \text { OLiquid superheating energy } \\ \square \text { Polynomial approach } & \Delta \text { Neural Net Fitting approach }\end{array}$

Figure 7. Results from the different methodologies vs the $\Delta \mathrm{P}$ experimental values.

\section{Example of application}

A cylindrical vessel with a volume of $80 \mathrm{~m}^{3}$, initially filled to $58 \%$ with liquid propane at room temperature $\left(20^{\circ} \mathrm{C}\right.$ and $\left.8.4 \mathrm{bar}\right)$, undergoes a BLEVE due to fire engulfment, when the content temperature is $50{ }^{\circ} \mathrm{C}$. The filling degree is $34 \%$ at the burst moment. Estimate overpressure $(\triangle \mathrm{P})$ at a distance of $100 \mathrm{~m}$. (Hemmatian et al., 2017b)

The MATLAB file for calculating the overpressure in $\mathrm{kPa}$ is:

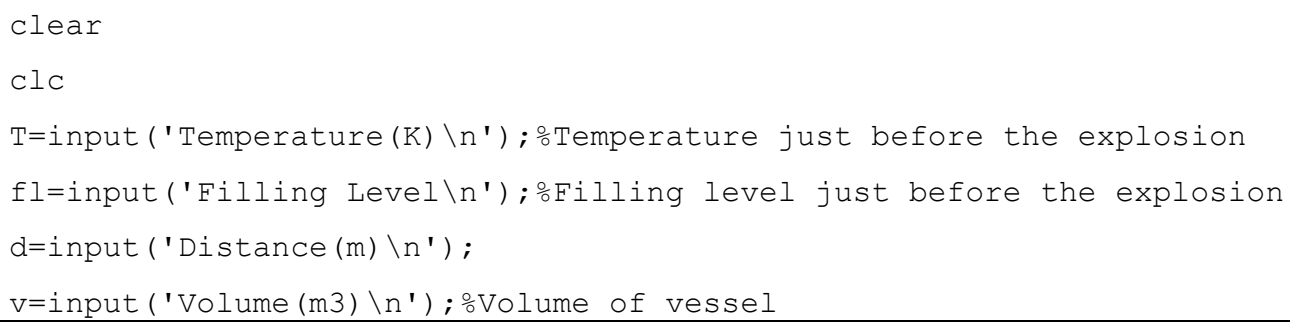




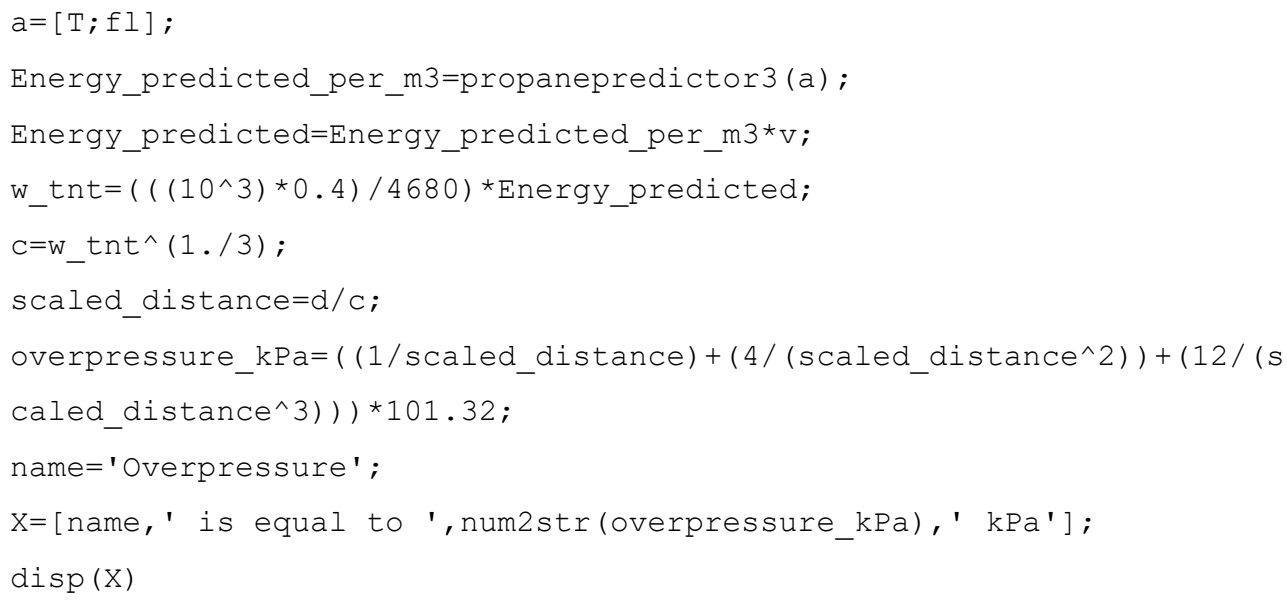

Here is the procedure for calculating the example and the result in $\mathrm{kPa}$ when running the previous MATLAB file:

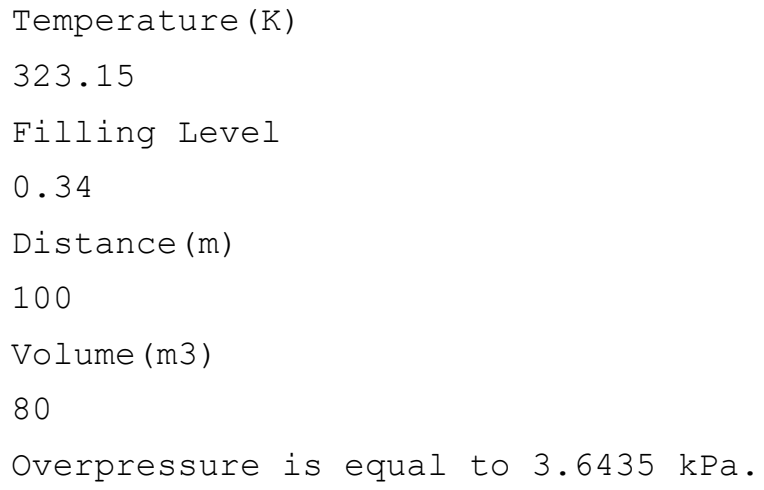

\section{Conclusion}

The Artificial Neural Network (ANN) approach was used to find the appropriate function between temperature and filling level as input data and the mechanical energy invested in creating the BLEVE peak overpressure as output data. Calculations were performed by assuming real gas behavior and adiabatic irreversible expansion, as this thermodynamic assumption provides more accurate and therefore realistic predictions than the others.

Two functions were generated for propane and butane by using ANN. The smallest MSE was given by three-layer Bayesian Regularization backpropagation algorithm with tangent sigmoid transfer function (tansig) at the hidden layer and linear transfer function (purelin) at output layer. 
For both substances, the designed networks regression $\mathrm{R}$ values were close to 1 , this meaning that there was a close correlation between outputs and targets.

To check the results from the ANN networks with two sets of experimental data from the literature, the corresponding peak overpressure values were obtained by applying the TNT equivalency method. According to the RMSE, calculated by various methodologies, the ANN networks showed a good level of performance (although there was a discrepancy in the near field for all the four applied methodologies). This, combined with its simplicity, allows obtaining quick and accurate results in an easy way. This methodology for calculating the mechanical energy is independent of substance's thermodynamic properties information (enthalpy, entropy, internal energy, etc), and it only requires the vessel filling level and temperature just before the explosion as input parameters to calculate the BLEVE mechanical energy as an output variable.

\section{Acknowledgments}

This research was partially funded by the Spanish Ministry of Economy and Competitiveness (projects CTM2014-57448-R and CTQ2017-85990-R, co-financed with FEDER funds), and by the Institut d'Estudis Catalans (Project PRO2018-S03). The authors thank the National Elites Foundation (Iran).

\section{Nomenclature}

$\begin{array}{ll}b & \text { Bias value } \\ \mathrm{b} & \text { Bios vector } \\ f & \text { Transfer function } \\ \mathrm{m} & \text { Mass }(\mathrm{kg}) \\ \mathrm{P}_{\text {rup }} & \text { Fialure pressure }(\mathrm{kPa}) \\ \mathrm{T} & \text { Temperature }(\mathrm{K}) \\ \mathrm{V} & \text { Volume }\left(\mathrm{m}^{3}\right) \\ w & \text { Weight value } \\ \mathrm{W} & \text { Weight matrix } \\ x_{\mathrm{i}} & \text { Input variable } \\ y & \text { Output variable } \\ \mathrm{Y} & \text { Output vector }\end{array}$




\section{References}

Beale, M. H., Hagan, M. T., Demuth, H. B., 2010. Neural network toolbox user's guide. The MathWorks Inc.

Birk, A. M., VanderSteen, J. D. J., 2006. On the transition from non-BLEVE to BLEVE failure for a $1.8 \mathrm{~m}^{3}$ propane tank. J Press Vessel Technol, 128(4), 648-655.

Birk, A. M., Davison, C., Cunningham, M. 2007. Blast overpressures from medium scale BLEVE tests. J Loss Prev Process Ind, 20(3), 194-206.

Brode, H. L.,1959. Blast wave from a spherical charge. Phys. Fluids, 2(2), 217-229.

Casal, J., Salla, J. M., 2006. Using liquid superheating energy for a quick estimation of overpressure in BLEVEs and similar explosions. J. Hazard Mater., 137(3), 1321-1327.

CCPS, 2010. Guidelines for Vapor Cloud Explosion, Pressure Vessel Burst, BLEVE, and Flash Fire Hazards, 2nd Edition (August 2010). Wiley Subscription Services, Inc., A Wiley Company.

Crowl, D. A., 1991. Using thermodynamic availability to determine the energy of explosion. Plant/Operations Progress, 10(3), 136-142.

Crowl, D. A., 1992. Using thermodynamic availability to determine the energy of explosion for compressed gases. Plant/Operations Progress, 11(2), 47-49.

Foresee, F. D., Hagan, M. T., 1997. Gauss-Newton approximation to Bayesian learning. In Proceedings of the 1997 international joint conference on neural networks (Vol. 3, pp. 19301935). Piscataway: IEEE.

Grossberg, S., 1982. Studies of Mind and Brain: Neural Principles of Learning, Perception, Development, Cognition, and Motor Control, Reidell Press, Boston, MA.

Hagan, M. T., Menhaj, M. B., 1994. Training feedforward networks with the Marquardt algorithm. IEEE transactions on Neural Networks, 5(6), 989-993.

Hebb, D.O. ,1949. The Organization of Behaviour: A Neuropsychological Theory, John Wiley, New York.

Hemmatian, B., Planas, E., Casal, J., 2015. Fire as a primary event of accident domino sequences: the case of BLEVE. Reliab. Eng. Syst. Safety, 139, 141-148.

Hemmatian, B., Planas, E., Casal, J., 2017a. Comparative analysis of BLEVE mechanical energy and overpressure modelling. Process Saf. Environ., 106, 138-149.

Hemmatian, B., Casal, J., Planas, E., 2017b. A new procedure to estimate BLEVE overpressure. Process Saf. Environ., 111, 320-325.

Hemmatian, B., Casal, J., Planas, E., Rashtchian, D., 2019. BLEVE: The case of water and a historical survey. J. Loss Prev. Process Ind, 57, 231-238. 
Hopfield, J. J., 1982. Neural networks and physical systems with emergent collective computational abilities. Proceedings of the national academy of sciences, 79(8), 2554-2558.

Johnson, D. M., Pritchard, M. J., Wickens, M. J., 1990. Large scale catastrophic releases of flammable liquids. Commission of the European Communities Report, Contract No. EV4T. 0014. $U K(H)$.

Laboureur, D., Heymes, F., Lapebie, E., Buchlin, J. M., Rambaud, P., 2014. BLEVE overpressure: multiscale comparison of blast wave modeling. Process Saf. Prog., 33(3), 274-284.

Lemmon, E. W., Huber, M. L., McLinden, M. O., 2007. REFPROP: Reference fluid thermodynamic and transport properties. NIST standard reference database, 23(8.0).

MacKay, D. J., 1992. A practical Bayesian framework for backpropagation networks. Neural Comput., 4(3), 448-472.

Møller, M. F. (1993). A scaled conjugate gradient algorithm for fast supervised learning. Neural Netw., 6(4), 525-533.

Nguyen, T. T., 1998. Earth-return path impedances of underground cables. Part 2: Evaluations using neural networks. IEE P-GENER TRANSM D, 145(6), 627-633.

Piñeiro, G., Perelman, S., Guerschman, J. P., Paruelo, J. M., 2008. How to evaluate models: observed vs. predicted or predicted vs. observed?. Ecol. Modell., 216(3-4), 316-322.

Planas-Cuchi, E., Salla, J. M., Casal, J., 2004. Calculating overpressure from BLEVE explosions. $J$ Loss Prev. Process Ind., 17(6), 431-436.

Planas, E., Pastor, E., Casal, J., Bonilla, J. M., 2015. Analysis of the boiling liquid expanding vapor explosion (BLEVE) of a liquefied natural gas road tanker: the Zarzalico accident. J. Loss Prev. Process Ind., 34, 127-138.

Prugh, R. W., 1991. Quantitative Evaluation of" Bleve" Hazards. J. Fire Prot. Eng., 3(1), 9-24.

Rumelhart, D. E., Hinton, G. E., Williams, R. J., 1986. Learning representations by backpropagating errors. Nature, 323(6088), 533.

Siddique, N., Adeli, H., 2013. Computational intelligence: synergies of fuzzy logic, neural networks and evolutionary computing. John Wiley \& Sons.

Smith, J. M., Van Ness, H. C., Abbott, M. M., 1996. McGraw Hill Chemical Engineering Series: Introduction to Chemical Engineering Thermodynamics. 632.

Ticknor, J. L., 2013. A Bayesian regularized artificial neural network for stock market forecasting. Expert Systems with Applications, 40(14), 5501-5506.

Widrow, B., Winter, R. G., Baxter, R. A. (1987, June). Learning phenomena in layered neural networks. In Proceedings of the IEEE First International Conference on Neural Networks (Vol. 2, pp. 411-430). 
Yadav, J.S., Yadav, M., Jain, A., 2014. Artificial neural network. International Journal of Scientific Research and Education. 1(06). 